\title{
Experimental Confirmation
} of Isotope Fractionation in Thiomolybdates Using lon

\section{Chromatographic Separation and \\ Detection by Multicollector ICPMS}

\author{
Journal Article \\ Author(s): \\ Kerl, Carolin F.; Lohmayer, Regina; Bura-Nakić, Elvira; Vance, Derek; Planer-Friedrich, Britta \\ Publication date: \\ 2017-03-07 \\ Permanent link: \\ https://doi.org/10.3929/ethz-b-000130385
}

Rights / license:

Creative Commons Attribution 4.0 International

Originally published in:

Analytical Chemistry 89(5), https://doi.org/10.1021/acs.analchem.6b04898 


\title{
Experimental confirmation of isotope fractionation in thiomolybdates using ion chromatographic separation and detection by multi-collector ICP-MS
}

\author{
Carolin F. Kerl, ${ }^{\dagger}$ Regina Lohmayer, ${ }^{\dagger}$ Elvira Bura-Nakić, ${ }^{\dagger}$ Derek Vance, ${ }^{\dagger}$ Britta Planer-Friedrich ${ }^{*} \dagger$ \\ ${ }^{\dagger}$ Department of Environmental Geochemistry, Bayreuth Center for Ecology and Environmental Research (BayCEER), \\ University of Bayreuth, Universitaetsstrasse 30, 95440 Bayreuth, Germany \\ ¥Institute of Geochemistry and Petrology, Department of Earth Sciences, ETH Zürich, Clausiusstrasse 25, 8092 Zürich, \\ Switzerland
}

\begin{abstract}
Molybdenum ${ }^{98} \mathrm{Mo} /{ }^{95} \mathrm{Mo}$ isotope ratios are a sediment paleo proxy for the redox state of the ancient ocean. Under sulfidic conditions, no fractionation between seawater and sediment should be observed if molybdate $\left(\mathrm{MoO}_{4}{ }^{2-}\right)$ is quantitatively transformed to tetrathiomolybdate $\left(\mathrm{MoS}_{4}{ }^{2-}\right)$ and precipitated. However, quantum mechanical calculations previously suggested that incomplete sulfidation could be associated with substantial fractionation. To experimentally confirm isotope fractionation in thiomolybdates, a new approach for determination of isotope ratios of individual thiomolybdate species was developed that uses chromatography (HPLC-UV) to separate individual thiomolybdates, collecting each peak and analyzing isotope ratios with multicollector inductively coupled plasma mass spectrometry (MC-ICP-MS). Using commercially available $\mathrm{MoO}_{4}{ }^{2-}$ and $\mathrm{MoS}_{4}{ }^{2-}$ standards, the method was evaluated and excellent reproducibility and accuracy were obtained. For species with longer retention times, complete chromatographic peaks had to be collected to avoid isotope fractionation within peaks. Isotope fractionation during formation of thiomolybdates could be experimentally proven for the first time in the reaction of $\mathrm{MoO}_{4}{ }^{2-}$ with 20 -fold or 50-fold excess of sulfide. The previously calculated isotope fractionation for $\mathrm{MoS}_{4}{ }^{2-}$ was confirmed and the result for $\mathrm{MoO}_{2} \mathrm{~S}_{2}{ }^{2-}$ was in the predicted range. Isotopic fractionation during $\mathrm{MoS}_{4}{ }^{2-}$ transformation with pressurized air was dominated by kinetic fractionation. Further optimization and online-coupling of the HPLC-MC-ICP-MS approach for determination of low concentrations in natural samples will greatly help to obtain more accurate species-selective isotope information.
\end{abstract}

\section{INTRODUCTION}

Sedimentary Mo and its isotopes are increasingly used as a paleo proxy for the redox state of the ancient ocean. ${ }^{1-4}$ Key to this application is the different behavior of Mo in oxic and anoxic environments. In oxic environments, the dominant aqueous Mo species is molybdate $\left(\mathrm{MoO}_{4}{ }^{2-}\right)$, which is highly soluble and inert. ${ }^{5}$ As a result, Mo is the most abundant transition metal in the dissolved pool of the oceans, where it is homogenously distributed, ${ }^{6,7}$ and has a long residence time of about $\sim 0.8 \mathrm{Ma} .{ }^{8,9}$ An important removal process in modern oceans is slow adsorption to particulate Mn-oxides in oxic conditions, which transfers Mo to sediment. ${ }^{8,10,11}$ Adsorption involves a $3 \%$ fractionation in the ${ }^{98} \mathrm{Mo} /{ }^{95} \mathrm{Mo}$ ratio, with the adsorbed Mo being lighter (throughout this paper we discuss Mo isotopes in terms of variations in the ${ }^{98} \mathrm{Mo} /{ }^{95} \mathrm{Mo}$ ratio, as $\delta^{98} \mathrm{Mo}$, with variations expressed in per mil (\%) deviations from NIST SRM 3134 set to $+0.25 \%$ by definition ${ }^{12}$ ). ${ }^{1,13-15}$ As a result, dissolved Mo in the oceans is $1.6 \%$ o heavier than the dominant riverine input, at $+2.3 \%$. ${ }^{6,13,16,17}$ In contrast, in euxinic waters where dissolved sulfide is present (such as the modern Black Sea) molybdate is progressively sulfidized, forming thiomolybdates $\left(\mathrm{MoO}_{4-\mathrm{x}} \mathrm{S}_{\mathrm{x}}{ }^{2-}, \mathrm{x}=1-4\right)$ via stepwise ligand exchange. ${ }^{18,19}$ At $\left[\mathrm{H}_{2} \mathrm{~S}\right]_{\text {aq }}$ above about 11 $\mu \mathrm{M}$, Mo is quantitatively transformed to tetrathiomolybdate $\left(\mathrm{MoS}_{4}{ }^{2-}\right) .{ }^{18}$ Tetrathiomolybdate is highly particle-reactive and removed near-quantitatively to sediment on very short timescales, ${ }^{18-25}$ leading to very high concentrations of authigenic Mo in reducing sediments. ${ }^{9,26}$ Because of the near-quantitative removal, the isotopic composition of the product must be identical to the starting $\mathrm{MoO}_{4}{ }^{2-}$ and sediment in locations like the Black Sea is isotopically almost identical to the open ocean Mo source. ${ }^{22-24}$

This simple bi-partite division of the modern Mo sinks, and the application of this view to the past, is complicated by a more complex behavior in intermediate situations. Isotopic fractionations from seawater $(-0.6$ to $-1.8 \%$ ) have been found in modern sediments deposited in conditions where dissolved sulfide occurs at low concentrations or intermittently in the water column, such as along the western margin of the Americas. ${ }^{14,}{ }^{27-30}$ In addition, the geological record of reducing sediments and rocks is increasingly turning up Mo isotope compositions that neither reflect the end-member oxic sink, nor record the seawater value of the end-member euxinic sink. ${ }^{2-4,31-36}$ Possible explanations of these intermediate fractionations are pore water processes and the involvement of sorption to Fe-oxides, for which fractionation relative to the aqueous phase has been shown experimentally to be smaller than for Mn oxides. ${ }^{13,15,29}$ However, these sediments are sulfidic in the sub-surface, and a second possibility is isotopic fractionation associated with incomplete sulfidation of $\mathrm{MoO}_{4}{ }^{2-}$. Theoretical calculations have suggested that each sulfidation step is associated with substantial fractionation, each step at around $+1.5 \%$ for $\delta^{98}$ Mo. ${ }^{37}$ Thus, if transformation to $\mathrm{MoS}_{4}{ }^{2-}$ is incomplete, and if there is 
different removal of the individual thiomolybdate species, there is potential to imprint substantial isotopic fractionations in sediments deposited beneath water columns that are anoxic or only moderately euxinic. This observation would go some way to explaining the observations of Mo in modern anoxic settings that are isotopically intermediate between fully euxinic and fully oxic. It could also explain the extremely light Mo isotopes in ancient sediments, that are ubiquitous but very difficult to interpret in a quantitative way. ${ }^{31,32}$

There has never been an experimental confirmation of the theoretically calculated isotopic fractionations between $\mathrm{MoO}_{4}{ }^{2-}$, intermediate thiomolybdates and $\mathrm{MoS}_{4}{ }^{2-}$. Here, we present the development and validation of a new method for measuring isotope ratios of individual thiomolybdate species by combining separation on high-performance liquid chromatography (HPLC-UV), collection of individual chromatographic peaks, and analysis by multicollector inductively coupled mass spectrometry (MC-ICPMS). We then used the method to track isotope fractionation during the formation of thiomolybdates from molybdate and sulfide and during the oxidative transformation of tetrathiomolybdate.

\section{MATERIAL AND METHODS}

\section{Stock solutions}

All stock solutions were prepared at the Environmental Geochemistry Group at the University of Bayreuth. A glovebox (COY, N $2 / \mathrm{H}_{2}$ 95/5\% (v/v)) was used for preparation of solutions. Stock solutions of ammonium molybdate $\left(\left(\mathrm{NH}_{4}\right)_{2} \mathrm{MoO}_{4}\right.$, Sigma-Aldrich), ammonium tetrathiomolybdate $\left(\left(\mathrm{NH}_{4}\right)_{2} \mathrm{MoS}_{4}\right.$, Sigma-Aldrich), and sodium sulfide nonahydrate $\left(\mathrm{Na}_{2} \mathrm{~S} \cdot 9 \mathrm{H}_{2} \mathrm{O}\right.$, Sigma-Aldrich) were prepared with $\mathrm{N}_{2}$-purged ultrapure water (Merck, Millipore, $18.2 \mathrm{~m} \Omega \cdot \mathrm{cm}, 7 \mathrm{ppb}$ total organic carbon at $25^{\circ} \mathrm{C}$ ). The $\mathrm{MoS}_{4}{ }^{2-}$ stock solution was filtered $(0.2 \mu \mathrm{m}$, celluloseacetate filter, Membrex or cellulose-nitrate, Whatmann) to remove insoluble particles, flash-frozen, and stored in liquid nitrogen immediately after preparation to ensure stability. ${ }^{38}$ Aliquots were thawed directly before starting the experiments to minimize alteration. All pipette tips (Sarstedt) and screw cap vials (1.5 mL glass (VWR), $2 \mathrm{~mL}$ PP (Hartenstein), and $10 \mathrm{~mL}$ PP (Sarstedt)) were acidcleaned with $3 \mathrm{M} \mathrm{HCl}$ (Grüssing) for $24 \mathrm{~h}$ and rinsed with ultrapure water.

\section{Speciation analysis}

The chromatographic method for Mo speciation analysis has been described in detail elsewhere. ${ }^{38}$ Briefly, it uses high-performance liquid chromatography (Merck Hitachi HPLC) coupled to UV/Vis detection. The chromatographic separation is based on ion-pairing using tetrabutylammonium hydroxide as ion pair reagent, a sodium carbonate buffer, and a gradient of $25-50 \%$ acetonitrile as eluent on a reversed-phase polymeric column (Dionex, IonPac NS1, $10 \mu \mathrm{m}, 250 \times 4 \mathrm{~mm}$ ). An example chromatogram from one of the experiments in the current study is shown in Fig. 1C. As described previously, ${ }^{38}$ $\mathrm{MoO}_{4}{ }^{2-}$ and $\mathrm{MoO}_{3} \mathrm{~S}^{2-}$ yielded two peaks, but could not be completely base-line separated. Besides $\mathrm{MoO}_{4}{ }^{2-}$ and the four thiomolybdates, a broad Mo peak was observed. This peak obviously consisted of different species which could not be base-line separated either. These peaks have previously been identified as polythiomolybdates $\left(\mathrm{Mo}_{\mathrm{x}} \mathrm{O}_{\mathrm{x}} \mathrm{S}_{\mathrm{x}}\left(\mathrm{S}_{\mathrm{x}}\right)_{\mathrm{x}}\right)$, which form through the polymerisation of thiomolybdates in aqueous solution, such as $\left[\mathrm{Mo}_{2} \mathrm{O}_{2} \mathrm{~S}_{2}\left(\mathrm{~S}_{2}\right)_{2}\right]^{2-} .{ }^{39}$

HPLC-UV chromatograms were obtained for all samples and $\mathrm{MoO}_{4}{ }^{2-}$ and $\mathrm{MoO}_{3} \mathrm{~S}^{2-}$ were integrated as two non-baseline-separated peaks. Molybdate and $\mathrm{MoS}_{4}{ }^{2-}$ were quantified in comparison to commercially available standards. Intermediate thiomolybdates were quantified according to the $\mathrm{MoO}_{4}{ }^{2-}$ standard with multiplication factors for the different UV absorption, ${ }^{38}$ but quantification of $\mathrm{Mo}_{\mathrm{x}} \mathrm{O}_{\mathrm{x}} \mathrm{S}_{\mathrm{x}}\left(\mathrm{S}_{\mathrm{x}}\right)_{\mathrm{x}}$ was not possible, as no multiplication factors exist. In addition to the HPLC-UV chromatograms, Mo concentrations of the individually collected fractions were measured by inductively coupled plasma mass spectrometry (ICP-MS) to obtain quantitative results for all species including $\mathrm{Mo}_{\mathrm{x}} \mathrm{O}_{\mathrm{x}} \mathrm{S}_{\mathrm{x}}\left(\mathrm{S}_{\mathrm{x}}\right)_{\mathrm{x}}$. When available, ICP-MS data was used for quantification of $\mathrm{Mo}_{\mathrm{x}} \mathrm{O}_{\mathrm{x}} \mathrm{S}_{\mathrm{x}}\left(\mathrm{S}_{\mathrm{x}}\right)_{\mathrm{x}}$, otherwise a semi-quantitative calibration was used. A linear regression line was calculated using the UV-signal versus the measured ICP-MS concentration of $\mathrm{Mo}_{\mathrm{x}} \mathrm{O}_{\mathrm{x}} \mathrm{S}_{\mathrm{x}}\left(\mathrm{S}_{\mathrm{x}}\right)_{\mathrm{x}}$. Polythiomolybdate concentrations of samples without available ICP-MS data were calculated according to this regression line. Since $\mathrm{MoO}_{4}{ }^{2-}$ and $\mathrm{MoO}_{3} \mathrm{~S}^{2-}$ were not baseline separated, they were collected in one fraction and total concentration is reported as sum of both species from the ICP-MS data.

\section{Analytical chromatography to obtain samples for} species-selective isotope analysis

To obtain individual thiomolybdates for isotope analysis, we used analytical chromatography and collected the chromatographic fractions for later off-line analysis by multi-collector inductively coupled plasma mass spectrometry (MC-ICP-MS). Chromatographic fractions were collected behind the UV-detector, which delivered online information for peak identification based on known retention times. The extra time for the sample to pass from the UV-detector to the end of the outlet tubing, where fractions were collected, was calculated and considered to ensure correct and complete peak collection. Each individual thiomolybdate peak was collected manually in acid cleaned polypropylene (PP) vials with screw caps (Sarstedt), sealed with parafilm (Pechiney) and stored at room temperature.

A significant concern was that chromatographic separation in itself might lead to an artefact isotope fractionation. We therefore compared the original stock solutions of $200 \mu \mathrm{M}$ $\mathrm{MoO}_{4}{ }^{2-}$ and $200 \mu \mathrm{M} \mathrm{MoS}_{4}{ }^{2-}$ to their respective chromatographic fraction in triplicates (CF R1, R2, R3). We further split both the $\mathrm{MoO}_{4}{ }^{2-}$ as well as the $\mathrm{MoS}_{4}{ }^{2-}$ chromatographic fraction in three parts and compared the results from the three parts to both the original solution and the triplicates from collection of the complete peak. The 
isotopic composition of particles, removed during $\mathrm{MoS}_{4}{ }^{2-}$ stock preparation by filtration, was also analyzed.

\section{Mo isotopic analysis}

All isotopic work was done at the ETH Zürich Isotope Geochemistry Group in "Class 100" cleanhoods, where PFA labware (Savillex) and trace metal clean acids (Merck AnalaR grade further purified by double sub-boiling distillation in teflon stills) were used for sample handling. First, Mo-containing chromatographic fractions collected from the HPLC-UV were evaporated on hot plates, to eliminate the eluent. Afterwards, they were re-dissolved with $500 \mu \mathrm{L} \mathrm{HNO}_{3}$ to remove any remaining organics, dried, and re-suspended in $5 \mathrm{~mL} 7 \mathrm{M} \mathrm{HCl}$. Molybdenum particles on filters were leached with $20 \mathrm{~mL} 0.6 \mathrm{M} \mathrm{HCl}$. Total Mo concentrations were measured by fast scanning magnetic-sector inductively coupled plasma mass spectrometry (ICP-MS, Element2, ThermoFinnigan) as $\mathrm{Mo}^{+}(\mathrm{m} / \mathrm{z}$ 98) with reference to an in house standard. Longterm reproducibility of Mo concentrations for secondary standard SLRS-5 is 10\% (2SD). Aliquots of samples taken for isotopic analysis were first spiked with ${ }^{97 / 100}$ Mo double spike in a 1:1 ratio to the Mo concentration of the sample to correct for mass discrimination. ${ }^{17,40}$ After equilibrating for $24 \mathrm{~h}$, Mo fractions were purified using $0.1 \mathrm{~mL}$ RE-resin-B (Triskem International, 50-100 $\mu \mathrm{m}$ ) loaded into homemade teflon columns. Prior to sample loading, the resin was cleaned with $2 \mathrm{~mL} 0.1 \mathrm{M} \mathrm{HCl}+0.3 \mathrm{M} \mathrm{HF}, 1 \mathrm{~mL}$ ultrapure water and conditioned with $2 \mathrm{~mL} 7 \mathrm{M} \mathrm{HCl}$. Matrix ions were separated by pre-elution with $5 \mathrm{~mL} 7 \mathrm{M} \mathrm{HCl}$ and 10 $\mathrm{mL} 1 \mathrm{M} \mathrm{HCl}$ before the Mo fraction was collected in 5.5 $\mathrm{mL} 0.2 \mathrm{M} \mathrm{HCl}$.

Isotope ratios were measured with MC-ICP-MS (Thermo Scientific Neptune Plus) using standard skimmer ("H") and sample cones. Samples were introduced to the instrument in $2 \% \mathrm{HNO}_{3}$ using an AridusII autosampler (CETAC) with a PFA nebulizer and spray chamber (CPI). Mo isotope ratios were measured relative to a CPI Mo standard, so that $\delta^{98} \mathrm{Mo}_{\text {sample }}=\left[{ }^{98} \mathrm{Mo} /{ }^{95} \mathrm{Mo}_{\text {sample }} /{ }^{98} \mathrm{Mo} /{ }^{95} \mathrm{Mo}_{\text {standard }}-1\right] \times 1000$. Verification of the accuracy and precision of the Mo double spike method was done via multiple analyses of mixtures of double spike and an in-house CPI Mo standard. Over the period of this study, standard/spike mixtures in a ratio of $0.5-2$ gave $\delta^{98} \mathrm{Mo}=-0.02 \pm 0.04 \%$ o $(2 \mathrm{SD}, \mathrm{n}=37)$ relative to NIST SRM $3134=+0.25$.

\section{Calculation of mass balance}

The mass balance in the experiments was assessed by comparing the measured starting Mo isotope composition $\delta^{98} \mathrm{Mo}_{\text {total }}$ with the calculated average in all fractions produced in the experiment:

$\sum \delta^{98} M o=\sum_{i=1}^{5} \delta_{i} \cdot \frac{M_{i}}{M_{\text {total }}}$

where $\delta_{\mathrm{i}}$ is the Mo isotopic composition of species $\mathrm{i}$ produced in the experiment, and $\mathrm{Mo}_{\mathrm{i}} / \mathrm{Mo}_{\text {total }}$ the mole fraction ( $\mathrm{m}$ in Eq. 2) of species i relative to the total Mo. The uncertainty on $\delta^{98}$ Mo is given by:

$f_{\text {error }}=\sqrt{\left(\frac{\partial\left(\sum \delta^{98} M o\right)}{\partial \delta}\right)^{2} \cdot \Delta \delta^{2}+\left(\frac{\partial\left(\sum \delta^{98} M o\right)}{\partial m}\right)^{2} \cdot \Delta m^{2}}($ Eq. 2$)$
Analytical error of ICP-MS measurements $(\Delta \mathrm{m})$ is taken into account as $10 \%$ (same technique as in ${ }^{41}$ ) and measured $2 \sigma$ values $(\Delta \delta)$ were used for $\delta^{98}$ Mo values in Eq. 2.

\section{Thiomolybdate (trans)formation experiments}

After method evaluation, isotope fractionation was tracked during formation of thiomolybdates from $\mathrm{MoO}_{4}{ }^{2-}$ and $\mathrm{HS}$ as well as during transformation of $\mathrm{MoS}_{4}{ }^{2-}$. All experimental work was done at the University of Bayreuth in the above described glovebox. For the formation experiment, the goal was to get a mixture of different thiomolybdates in equilibrium where each thiomolybdate had high enough concentrations to be measurable by MCICP-MS. Since we knew from a previous experiment that a 10 -fold excess of sulfide would dominantly yield $\mathrm{MoOS}_{3}$ but little $\mathrm{MoS}_{4}{ }^{2-},{ }^{38}$ here we used $250 \mu \mathrm{M}\left(\mathrm{NH}_{4}\right)_{2} \mathrm{MoO}_{4}$ with a 20- and 50-fold excess of $\mathrm{Na}_{2} \mathrm{~S}$. The starting $\mathrm{pH}$ (WTW multimeter (Multi 340i) with WTW SenTix 41-3 pH electrode) was adjusted to 7.3 with $\mathrm{HCl}$ (Grüssing) because we previously observed the fastest reaction kinetics at that pH. ${ }^{38}$ We used screw-capped $50 \mathrm{~mL}$ PFA beakers (Savillex) and allowed solutions to equilibrate during preparation and sampling with the glovebox atmosphere, which resulted in some sulfide loss and a corresponding $\mathrm{pH}$ increase up to $\mathrm{pH}$ of approximately 9 . At that $\mathrm{pH}$, we had previously observed thiomolybdate formation and transformation to be almost negligible. ${ }^{38}$ The $\mathrm{pH}$ increase therefore served as a stopper to the formation reaction, "freezing" in a mixture of species, and preventing the transformation of all $\mathrm{MoO}_{4}{ }^{2-}$ to end-member $\mathrm{MoS}_{4}{ }^{2-}$. Samples were taken from original stock, after $1 \mathrm{~h}$ (day 0) and 3, 8, and 13 days for Mo speciation analysis as well as for analysis of total and/or species-selective Mo isotope signatures. Species were separated with HPLC-UV immediately after sampling. In the transformation experiment, $200 \mu \mathrm{M}\left(\mathrm{NH}_{4}\right)_{2} \mathrm{MoS}_{4}$ solution was purged in an open vessel outside the glovebox with 1 bar pressurized air, taking samples for speciation after 0.5, 1, 2.5, 5, 7.5, 10, 24, $33,50,72.5$ and $79 \mathrm{~h}$ and samples for isotope analysis after 33,50 and $72.5 \mathrm{~h}$.

\section{RESULTS AND DISCUSSION}

\section{Method evaluation}

To test whether chromatography can separate Mo species without isotope fractionation, commercially available standards $\mathrm{MoO}_{4}{ }^{2-}$ and $\mathrm{MoS}_{4}{ }^{2-}$ were used. There were no significant differences in isotope composition between the original $\mathrm{MoO}_{4}{ }^{2-}$ stock solution $(-0.30 \pm 0.03 \%$ ) and the complete $\mathrm{MoO}_{4}{ }^{2-}$ peaks collected after chromatographic

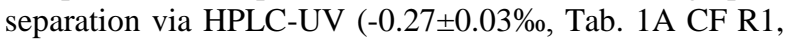
R2, R3). Moreover, no isotope fractionation occurred within the chromatographic peak, when split into three parts (Fig. 1A), and the calculated mass balance $(-0.29 \pm 0.10 \%)$ was in agreement with the original $\mathrm{MoO}_{4}{ }^{2-}$ stock solution (Tab. 1A part I-III).

The original stock solution of $\mathrm{MoS}_{4}{ }^{2-}$ showed an isotope signature of $0.09 \pm 0.02 \%$. Surprisingly, in a first test, the chromatographically separated $\mathrm{MoS}_{4}{ }^{2-}$ triplicates yielded higher values $(0.25 \pm 0.03 \%$, Tab. $1 \mathrm{~A} \mathrm{CF} \mathrm{R1,} \mathrm{R2,} \mathrm{R3).}$ Furthermore, isotope fractionation occurred within the split 
$\mathrm{MoS}_{4}{ }^{2-}$ peak with the heavy isotopes eluting first $(0.55 \%$ in the first part compared to $0.08 \%$ in the third part, Tab. $1 \mathrm{~A}$ $\mathrm{CF}$ part I-III, Fig. 1B). However, the calculated mass balance (Eq. 1) over all three peak splits was in accordance with the measured isotope fractionation of the total peak $(0.23 \pm 0.17$ compared to $0.25 \pm 0.03 \%)$. The isotope fractionation within the peak might be explained by the longer retention time of $\mathrm{MoS}_{4}{ }^{2-}$ (12 min compared to 5 min for $\mathrm{MoO}_{4}{ }^{2-}$ ). Faster elution of heavy isotopes when using different anion-exchange resins has been attributed before to equilibrium isotope fractionation between dissolved and resin-bound complexes ${ }^{42-44}$. However, this has not been investigated so far for ion-pairing chromatography. Analytical problems or sample loss can be excluded as a reason for isotope fractionation because the calculated mass balance of the split peaks was in accordance with the chromatographically separated peak. The conclusion to draw is that for obtaining unbiased isotope ratios, the complete chromatographic peaks must be collected because thiomolybdates with longer retention time can show isotope fractionation within the chromatographic peak.

Table 1. Mo isotope values from analyzing $\mathrm{MoO}_{4}{ }^{2-}$ and $\mathrm{MoS}_{4}{ }^{2-} \quad$ solutions directly ("original"), after chromatographic separation and collection of the whole peak (CF with three replicates $\mathrm{R} 1, \mathrm{R} 2, \mathrm{R} 3)$ and collection of 3 equal peak splits (CF part I-III). A: flash-frozen $\mathrm{MoS}_{4}{ }^{2-}$ ; B: freshly prepared $\mathrm{MoS}_{4}{ }^{2-}$

A:

\begin{tabular}{|c|c|c|c|c|}
\hline \multirow[t]{2}{*}{ Sample } & \multicolumn{2}{|c|}{$200 \mu \mathrm{M} \mathrm{MoO}_{4}{ }^{2-}$} & \multicolumn{2}{|c|}{$200 \mu \mathrm{M} \mathrm{MoS}_{4}^{2-}$} \\
\hline & $\begin{array}{c}\delta^{98} \mathrm{Mo} \\
(\%)\end{array}$ & $2 \sigma$ & $\begin{array}{c}\delta^{98} \mathrm{Mo} \\
(\% о)\end{array}$ & $2 \sigma$ \\
\hline original & -0.30 & \pm 0.03 & 0.09 & \pm 0.02 \\
\hline CF R1 & -0.29 & \pm 0.02 & 0.25 & \pm 0.02 \\
\hline CF R2 & -0.24 & \pm 0.02 & 0.25 & \pm 0.03 \\
\hline CF R3 & -0.27 & \pm 0.02 & 0.25 & \pm 0.03 \\
\hline mean $\mathrm{CF}$ & -0.27 & \pm 0.03 & 0.25 & \pm 0.03 \\
\hline CF part I & -0.28 & \pm 0.05 & 0.55 & \pm 0.05 \\
\hline CF part II & -0.29 & \pm 0.02 & 0.29 & \pm 0.03 \\
\hline CF part III & -0.30 & \pm 0.02 & 0.08 & \pm 0.03 \\
\hline mean CF & -0.29 & \pm 0.10 & 0.23 & \pm 0.17 \\
\hline \multicolumn{5}{|l|}{ B: } \\
\hline \multirow[t]{2}{*}{ Sample } & \multicolumn{2}{|c|}{$200 \mu \mathrm{M} \mathrm{MoS} 4^{2-}$} & \multicolumn{2}{|c|}{ Filter } \\
\hline & $\begin{array}{c}\delta^{98} \mathrm{Mo} \\
(\% o)\end{array}$ & $2 \sigma$ & $\begin{array}{c}\delta^{98} \mathrm{Mo} \\
(\% \text { o })\end{array}$ & $2 \sigma$ \\
\hline original & -0.01 & \pm 0.02 & & \\
\hline CF R1 & -0.03 & \pm 0.01 & -0.02 & \pm 0.02 \\
\hline CF R2 & 0.02 & \pm 0.02 & -0.04 & \pm 0.02 \\
\hline CF R3 & 0.03 & \pm 0.02 & -0.05 & \pm 0.02 \\
\hline mean CF & 0.01 & \pm 0.03 & -0.04 & \pm 0.02 \\
\hline
\end{tabular}

To further elucidate the observed differences between isotope signatures of the $\mathrm{MoS}_{4}{ }^{2-}$ solution before $(0.09 \pm 0.02 \%$ o $)$ and after $(0.25 \pm 0.03 \%$ ) chromatographic separation, we did a second test with a $\mathrm{MoS}_{4}{ }^{2-}$ standard, which in contrast to the first one was freshly prepared and directly used, not flash-frozen in between. The solution this time showed a perfect match between original solution ($0.01 \pm 0.02 \%$ ) and triplicates of the chromatographic fraction $(0.01 \pm 0.03 \%$, Tab. $1 \mathrm{~B})$. The reason for the discrepancy between the two tests remains unclear. Different amounts of undissolved particles in different $\mathrm{MoS}_{4}{ }^{2-}$ standards, inducing different isotope fractionation, can be excluded as the reason, since the isotope signature of Mo on the filters used to separate undissolved particles prior to analysis was identical to the stock solution (Tab. 1B). Small amounts of other dissolved species contained in the $\mathrm{MoS}_{4}{ }^{2-}$ standard as impurities could potentially also contribute to the observed differences. Molybdate, $\mathrm{MoO}_{3} \mathrm{~S}^{2-}$ , and $\mathrm{MoO}_{2} \mathrm{~S}_{2}{ }^{2-}$ were not detected in chromatograms, but a small impurity of $\mathrm{MoOS}_{3}{ }^{2-}$ was visible. As the concentration of $\mathrm{MoOS}_{3}{ }^{2-}$ was too small for quantifying $\delta^{98} \mathrm{Mo}$, a fraction of $\mathrm{MoOS}_{3}{ }^{2-}+\mathrm{MoS}_{4}{ }^{2-}$ was analyzed but showed the same $\delta^{98} \mathrm{Mo}$ as the pure $\mathrm{MoS}_{4}{ }^{2-}$ peak. We therefore also exclude any influence of intermediate thiomolybdates. What we did observe only in flash-frozen and thawed, but never in freshly prepared $\mathrm{MoS}_{4}{ }^{2-}$ standards, were additional peaks of Mo species eluting after $\mathrm{MoS}_{4}{ }^{2}$, peaks that have previously been identified as $\mathrm{Mo}_{\mathrm{x}} \mathrm{O}_{\mathrm{x}} \mathrm{S}_{\mathrm{x}}\left(\mathrm{S}_{\mathrm{x}}\right)_{\mathrm{x}}$ 39. This would require $\mathrm{Mo}_{\mathrm{x}} \mathrm{O}_{\mathrm{x}} \mathrm{S}_{\mathrm{x}}\left(\mathrm{S}_{\mathrm{x}}\right)_{\mathrm{x}}$ formed from $\mathrm{MoS}_{4}{ }^{2-}$ to have a lighter isotope signature, but as the concentrations were too low for quantification, we could not finally confirm this. To avoid any isotope fractionation by artifacts from flash-freezing Mo-containing solutions, all samples in the following experiments were analyzed immediately.
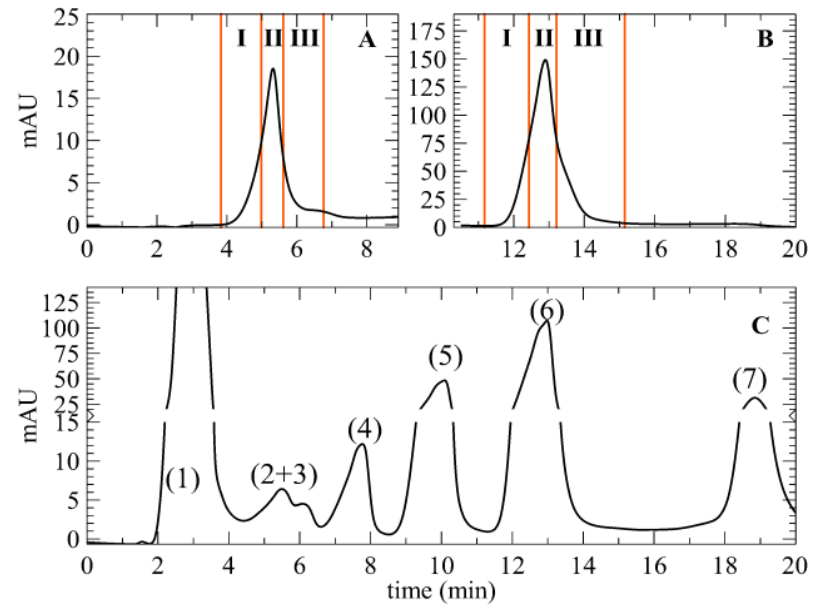

Figure 1. A: $\mathrm{MoO}_{4}{ }^{2-}$ peak split into 3 parts. B: $\mathrm{MoS}_{4}{ }^{2-}$ peak split into 3 parts. C: Example chromatogram of Mo:S 1:20 at day 3 showing (1) sulfide, (2+3) $\mathrm{MoO}_{4}^{2-} \& \mathrm{MoO}_{3} \mathrm{~S}^{2-}$, (4) $\mathrm{MoO}_{2} \mathrm{~S}_{2}^{2-}(5) \mathrm{MoOS}_{3}{ }^{2-}$, (6) $\mathrm{MoS}_{4}^{2-}$, (7) $\mathrm{Mox}_{\mathrm{x}} \mathrm{O}_{\mathrm{x}} \mathrm{S}_{\mathrm{x}}\left(\mathrm{S}_{\mathrm{x}}\right)_{\mathrm{x}}$. 


\section{Isotope fractionation during formation of thiomolybdates}

As described in the methods section, the transformation experiment consisted of a dynamic phase (day 0-3) and an equilibration phase (day 3-13). During the dynamic phase, sulfide concentrations decreased to about $86.5 \pm 0.5 \%$ of the initial concentration in both experiments and thereafter remained constant from day 3 to 13 (Tab. $1 \mathrm{SI}$ ). The $\mathrm{pH}$ correspondingly increased to 9.0 and 9.5 in the experiments with a 20-fold and a 50-fold excess of sulfide, respectively (Tab. 1 SI). Mo speciation analysis showed immediate formation of $\mathrm{MoO}_{3} \mathrm{~S}^{2-}$ and $\mathrm{MoO}_{2} \mathrm{~S}_{2}{ }^{2-}$ with maximum concentrations after $1 \mathrm{~h} \mathrm{(44}$ and $43 \%$, respectively) in the 1:20 experiment (Fig. 2A). With further reaction, these concentrations declined as $\mathrm{MoOS}_{3}{ }^{2-}$ and $\mathrm{MoS}_{4}{ }^{2-}$ became the dominant species (both $34 \%$ after 3 days). No $\mathrm{MoO}_{4}{ }^{2-}$ was present after $1 \mathrm{~h}$ but within 13 days concentrations increased to $17 \%$ (no $\mathrm{MoO}_{4}{ }^{2-}$ occurred in HPLC-UV chromatogramm after $1 \mathrm{~h}$ ). Polythiomolybdate formation was observed until day 7 and concentrations remained stable afterwards, at around $8 \%$. Measured total Mo concentrations were constant throughout the experiment. Though the general pattern of Mo speciation is very similar for the 1:50 experiment (Fig. 2B), there are two interesting differences. Throughout the experiment, the share of $\mathrm{MoOS}_{3}{ }^{2-}$ remained higher than that of $\mathrm{MoS}_{4}{ }^{2-}$ at 50 -fold excess sulfide, whereas at 20-fold excess equal concentrations were reached. The reason is a faster increase of the $\mathrm{pH}$ to a slightly higher value at 50 -fold excess of sulfide (at day 3: $\mathrm{pH} 9.1$ compared to $\mathrm{pH} 8.2$ in the 1:20 experiment), which stopped the formation reaction before much $\mathrm{MoS}_{4}{ }^{2-}$ could form. The second interesting difference is a higher share of $\mathrm{Mo}_{\mathrm{x}} \mathrm{O}_{\mathrm{x}} \mathrm{S}_{\mathrm{x}}\left(\mathrm{S}_{\mathrm{x}}\right)_{\mathrm{x}}$, which indicates that these species are not the end member of a reaction from $\mathrm{MoO}_{4}{ }^{2-}$ to $\mathrm{MoS}_{4}{ }^{2-}$, but must form intermittently, e.g. from $\mathrm{MoS}_{2} \mathrm{O}_{2}{ }^{2-}$ as suggested previously. ${ }^{39}$
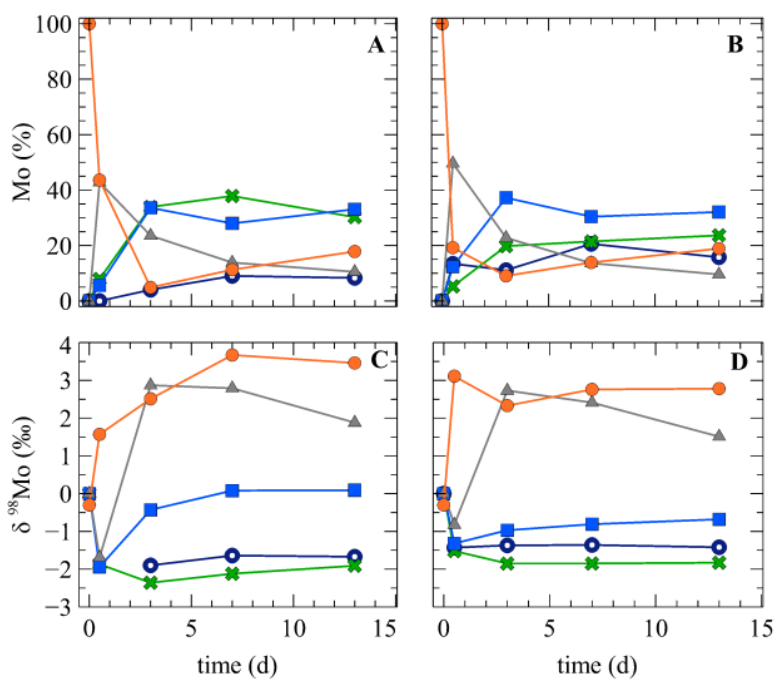

$\begin{array}{lll}-\mathrm{MoO}_{4}^{2-} / \mathrm{MoO}_{3} \mathrm{~S}^{2-} & -\mathrm{m} \mathrm{MoOS}_{3}^{2-} \\ -\mathrm{MoO}_{2} \mathrm{~S}_{2}^{--} & -\mathrm{MoS}_{4}^{2-}\end{array}$

$\rightarrow \mathrm{Mo}_{\mathrm{x}} \mathrm{O}_{\mathrm{x}} \mathrm{S}_{\mathrm{x}}\left(\mathrm{S}_{\mathrm{x}}\right)_{\mathrm{x}}$

Figure 2. Mo-species separated with HPLC-UV and chromatographic fractions analyzed with ICP-MS in \% (A,B) and $\delta{ }^{98} \mathrm{Mo}$ values for Mo-species measured with MC-ICP-MS in \%o (C,D). A+C: $250 \mu \mathrm{M} \mathrm{MoO}_{4}{ }^{2-}+5 \mathrm{mM} \mathrm{HS}^{-}(\mathrm{Mo:S} 1: 20)$, $\mathrm{B}+\mathrm{D}: 250 \mu \mathrm{M} \mathrm{MoO}_{4}{ }^{2-}+12.5 \mathrm{mM} \mathrm{HS}^{-}$(Mo:S 1:50).

Figure 2C,D shows the $\delta^{98} \mathrm{Mo}$ values for the individual thiomolybdate species from the above described experiments. As expected, in both experiments, $\mathrm{MoO}_{4}{ }^{2-}$ $+\mathrm{MoO}_{3} \mathrm{~S}^{2-}$ became enriched in heavier isotopes with the reaction proceeding until day $7\left(\delta^{98} \mathrm{Mo}=+3.67\right.$ in the $1: 20$ and $+2.76 \%$ in the $1: 50$ experiment, Tab. 1, SI). A prominent feature of both experiments is that the initial $\mathrm{MoO}_{2} \mathrm{~S}_{2}{ }^{2-}$ had a very light isotopic composition (-1.69 and $-0.82 \%$ ), which became heavier through the experiment $(+1.88$ and $+1.51 \%$ at day 13$)$. Trithiomolybdate showed a much more subtle version of the same behavior. Tetrathiomolybdate and $\mathrm{Mo}_{\mathrm{x}} \mathrm{O}_{\mathrm{x}} \mathrm{S}_{\mathrm{x}}\left(\mathrm{S}_{\mathrm{x}}\right)_{\mathrm{x}}$ were similarly enriched in light isotopes initially, and stayed relatively stable throughout the experiments. By the end of both experiments the $\delta^{98} \mathrm{Mo}$ decreased in the following order: $\mathrm{MoO}_{4}{ }^{2-}+\mathrm{MoO}_{3} \mathrm{~S}^{2-}>\mathrm{MoO}_{2} \mathrm{~S}_{2}{ }^{2-}>\mathrm{MoOS}_{3}{ }^{2-}>\mathrm{Mo}_{\mathrm{x}} \mathrm{O}_{\mathrm{x}} \mathrm{S}_{\mathrm{x}}\left(\mathrm{S}_{\mathrm{x}}\right)_{\mathrm{x}} \sim$ $\mathrm{MoS}_{4}{ }^{2-}$.

\section{Comparison with theoretical calculations}

For a better comparison between the experimental data and literature values, $\Delta^{98}$ Mo values were calculated (Tab. $2, \mathrm{SI}$ ). Tossell ${ }^{37}$ used two different quantum mechanical models to calculate equilibrium isotope fractionations between thiomolybdates $\Delta_{0-2}$ and $\Delta_{0-4}\left(=\delta^{98} \mathrm{Mo} \mathrm{MoO}_{4}{ }^{2-}-\delta^{98} \mathrm{Mo}\right.$ $\mathrm{MoO}_{2} \mathrm{~S}_{2}{ }^{2-}$ and $\delta^{98} \mathrm{Mo} \mathrm{MoO}_{4}{ }^{2-}-\delta^{98} \mathrm{Mo} \mathrm{MoS}_{4}{ }^{2-}$ ). Using a hybrid Hartree-Fork - discrete Fourier transformation (B3LYP) values are $\Delta_{0-2}=2.4 \%$ and $\Delta_{0-4}=5.4 \%$, using only a Hartree-Fork method they are $\Delta_{0-2}=3.3 \%$ and $\Delta_{0 \text { - }}$ ${ }_{4}=6.75 \%$. The differences of 0.9 and $1.35 \%$, respectively, imply that there are some uncertainties in these theoretical calculations. Tossell ${ }^{37}$ concluded that the B3LYP values are the more accurate ones.

Table 2. Mass balance and Mo recoveries ( $\sum \mathrm{Mo}$ fractions/total Mo) of thiomolybdate formation experiments with comparison between calculated mass balance (Eq. 1) and measured unfractionated sampled.

\begin{tabular}{|c|c|c|c|c|c|}
\hline Time (days) & 0 & 3 & 7 & & 13 \\
\hline Experiment & $1: 20$ & & & & \\
\hline Mass balance & $\delta^{98} \mathrm{Mo} \mathrm{( \% o)}$ & & & & \\
\hline calculated $^{1}$ & $-0.30 \pm 0.25$ & $|-0.22 \pm 0.16|$ & $|-0.13 \pm 0.18|$ & 0.13 & \pm 0.15 \\
\hline measured & $-0.28 \pm 0.02$ & $-0.32 \pm 0.02$ & $-0.28 \pm 0.02$ & -0.29 & \pm 0.03 \\
\hline Recovery (\%) & $79 \pm 16$ & $91 \pm 16$ & $88 \pm 16$ & 80 & \pm 16 \\
\hline
\end{tabular}

Experiment 1:50

Mass balance $\delta^{98} \mathrm{Mo} \mathrm{( \% o)}$

\begin{tabular}{c|cc|cc|cc|cc} 
calculated $^{1}$ & -0.25 & \pm 0.16 & -0.05 & \pm 0.15 & -0.21 & \pm 0.13 & -0.20 & \pm 0.11 \\
measured $^{-0.29}$ & \pm 0.02 & -0.30 & \pm 0.03 & -0.30 & \pm 0.03 & -0.28 & \pm 0.02 \\
\hline Recovery $(\%)$ & 79 & \pm 16 & 66 & \pm 16 & 82 & \pm 16 & 85 & \pm 16 \\
\hline
\end{tabular}

${ }^{1}$ Uncertainties calculated using Eq. 2

At the end of our experiments (day 13), $\Delta_{0-2}$ was 1.58 and $1.27 \%$ while $\Delta_{0-4}$ was 5.37 and $4.61 \%$ in the $1: 20$ and 1:50 experiment, respectively (the index zero in $\Delta_{0-2}$ and $\Delta_{0-4}$ 
represents the sum of $\mathrm{MoO}_{4}{ }^{2-}+\mathrm{MoO}_{3} \mathrm{~S}^{2-}$, as the peaks coeluted). The lower value at 1:50 might be explained with the above described pre-mature stopping of the thiomolybdate formation reaction resulting in less $\mathrm{MoS}_{4}{ }^{2-}$ and a higher share of $\mathrm{Mo}_{\mathrm{x}} \mathrm{O}_{\mathrm{x}} \mathrm{S}_{\mathrm{x}}\left(\mathrm{S}_{\mathrm{x}}\right)_{\mathrm{x}}$. However, given the proportion of $\mathrm{MoO}_{3} \mathrm{~S}^{2-}$ in the co-eluted peak (about $15 \%$ at day 13) and assuming that the isotope ratio for the mono species in the mixture must be greater than the corresponding dithio species, then the maximum possible impact of the mono species is $0.2-0.3 \%$. Therefore, a pure $\mathrm{MoO}_{4}{ }^{2-}$ peak would be, at most, $0.2-0.3 \%$ higher in $\delta^{98} \mathrm{Mo}$. Thus, our measurements at day $13(1: 20)$ suggest that $\Delta_{0}$ ${ }_{2}=1.4-1.7 \%$ while $\Delta_{0-4}=5.0-5.3 \%$. The latter is very close to the value of $5.4 \%$ calculated by Tossell ${ }^{37}$ using B3LYP. The experimental $\Delta_{0-2}$ value is $0.7-1 \%$ o less than the $2.4 \%$ calculated by Tossell 37 using the B3LYP method. However, $\mathrm{MoO}_{2} \mathrm{~S}_{2}{ }^{2-}$ is the most dynamic pool in its abundance and isotopic composition. $\delta^{98}$ Mo still evolves significantly between days 7 and 13 and the small size of this pool (6-7\% of the total) makes it the most sensitive to minor continuing isotopic exchanges with larger pools, even if those larger pools are themselves stable through time. Clearly, our experimental findings are qualitatively consistent with the calculations for $\mathrm{MoO}_{2} \mathrm{~S}_{2}{ }^{2-}$ and $\mathrm{MoS}_{4}{ }^{2-}{ }^{37}$, as well as the assumptions that each successive substitution of sulfur leads to an isotopically lighter species at equilibrium. ${ }^{23,24,45}$

Overall, we were able to show, for the first time experimentally, that isotope fractionation occurs among different thiomolybdate species and that our experimental results for the two end-members, $\mathrm{MoO}_{4}{ }^{2-}$ and $\mathrm{MoS}_{4}{ }^{2-}$, are in line with previously calculated values, while intermediate thiomolybdates will likely require consideration of kinetic isotope fractionation.

\section{Mass balance}

In order to evaluate recovery of isotope ratios, mass balances and analytical errors were calculated according to equation 1 and 2. The mass balances were in good agreement with the isotopic composition of the unfractionated sample except for Mo to $S$ ratios 1:50 at day 3 and Mo to $S$ ratios 1:20 at day 13 (Tab. 2). The unexpected light $\delta^{98} \mathrm{Mo}$ for $\mathrm{MoO}_{4}{ }^{2-} / \mathrm{MoO}_{3} \mathrm{~S}^{2-}$ at day 3 (1:50) could be linked to an observed problem during sample injection for chromatography (compare SI Tab.1, where recovery of total Mo $(66 \%)$ and sulfide $(56 \%)$ are also too low), but no obvious problem occurred at day 13 (1:20). Despite the problem with these two mass balances, the good overall agreement showed that no sample was lost during chromatographic separation and further preparation for MC-ICP-MS measurement. Especially precipitation, which would influence the results significantly, could be excluded in our experiments.

Total Mo recoveries in ng (Tab.1 SI) determined as the sum of Mo in the individual chromatographic fractions versus total Mo in the non-separated solution averaged about $83 \pm 16 \%$ (Tab. 2). The relatively high standard deviation is explained by the fact that the sum of Mo consists of 5 individual species, which are all determined with a certain analytical error. The systematic offset from $100 \%$ recovery indicates loss. The reasons for such loss could be slight differences in the exact sample volumes taken (nominal 100 $\mu \mathrm{L}$ ), because one (for Mo species) was injected by the HPLC autosampler syringe, the other (for total Mo) pipetted directly from solution. Another source of loss is incomplete sample transfer during evaporation, redissolution, drying, or resuspension before analysis by MC-ICP-MS, which would be multiplied by a factor 5 for the 5 Mo species treatments versus 1 total Mo treatment. It is important to note that such losses have no impact at all on measured Mo isotope composition. Considering the number of species involved and sample handling between two non-coupled instruments, with all the necessary cleanup steps, the recovery is good.

\section{Isotope fractionation during tetrathiomolybdate transformation}

Speciation and isotope fractionation were also investigated during transformation of thiomolybdates by purging $\mathrm{MoS}_{4}{ }^{2-}$ with air for 79 hours. Tetrathiomolybdate was completely transformed to $\mathrm{MoO}_{4}{ }^{2-}$ after $79 \mathrm{~h}$ (Fig. 3A). Intermediate thiomolybdates contributed up to $12 \%$ after $50 \mathrm{~h}(6 \%$ and $5 \%$ of $\mathrm{MoO}_{2} \mathrm{~S}_{2}{ }^{2-}$ and $\mathrm{MoOS}_{3}{ }^{2-}$, respectively, Tab. $3 \mathrm{SI}$ ) and $\mathrm{MoO}_{4}{ }^{2-}$ started to be the dominant species after $55 \mathrm{~h}$. Polythiomolybdates did not account for more than $2 \%$ at any time. Isotope ratios were measured for selected data points only, as concentrations of formed species were too low at the beginning of the transformation. As the oxidation proceeded, $\delta^{98}$ Mo values for $\mathrm{MoS}_{4}{ }^{2-}(2.18 \%$ after $72.5 \mathrm{~h}$, Tab. $4 \mathrm{SI})$ became heavier, while the formed $\mathrm{MoO}_{4}{ }^{2-}$ $\left(0.07 \%\right.$ ) approached the starting value of $\mathrm{MoS}_{4}{ }^{2-}(0.09 \%$, Fig. 3B). Differences among the formed intermediate species were small, but all species became enriched in heavy isotopes with ongoing oxidation. As the measurements for Mo concentrations as well as isotope ratios were close to the detection limit, mass balances were calculated from HPLC-UV and ICP-MS data to minimize uncertainties.

The mass balance (Eq .1) was closed for all data points using species distribution obtained by HPLC-UV, but could not be completely closed using ICP-MS data for 33 and 50 h (Tab. 3). Considering that the measured concentrations were close to the detection limit in both measurement techniques, the unclosed mass balance might be due to analytical uncertainties rather than due to precipitation or sample loss. The fact that, after complete transformation, the isotopic signature of the initial $\mathrm{MoS}_{4}{ }^{2-}$ was obtained in $\mathrm{MoO}_{4}{ }^{2-}(0.09$ compared to $0.07 \%$ o), clearly confirms that no loss or contamination occurred during the experiment. 
Table 3. Mass balance of thiomolybdate transformation experiment with comparison between calculated mass balance (Eq. 1) using ICP-MS and HPLC-UV data and measured initial $\mathrm{MoS}_{4}{ }^{2-}$.

\begin{tabular}{|c|c|c|c|c|}
\hline \multicolumn{5}{|c|}{ Mass balance $\delta^{98}$ Mo (\%o) } \\
\hline Time (h) & 33 & & 50 & 72.5 \\
\hline Calc. ICP-MS & $0.34 \pm 0.16^{1}$ & 0.37 & $\pm 0.15^{1}$ & $0.25 \pm 0.28^{1}$ \\
\hline Calc. HPLC-UV & $0.25 \pm 0.16^{1}$ & 0.25 & $\pm 0.15^{1}$ & $0.15 \pm 0.28^{1}$ \\
\hline $\mathrm{MoS}_{4}^{2-}$ at start & $0.09 \pm 0.02$ & 0.09 & \pm 0.02 & \pm 0.02 \\
\hline
\end{tabular}

${ }^{1}$ Uncertainties calculated using Eq. 2

All transformation products formed during the experiment were isotopically lighter than the respective $\mathrm{MoS}_{4}{ }^{2-}$ and the residual reactant became depleted in the light isotope through the experiment. The simplest interpretation of this result is that the isotope fractionations seen are kinetic. This conclusion is consistent with the rapidity of the near complete transformation of $\mathrm{MoS}_{4}{ }^{2-}$ to $\mathrm{MoO}_{4}{ }^{2-}(\sim 80 \mathrm{~h})$, and with the fact that the experimental products are lighter than reactants.

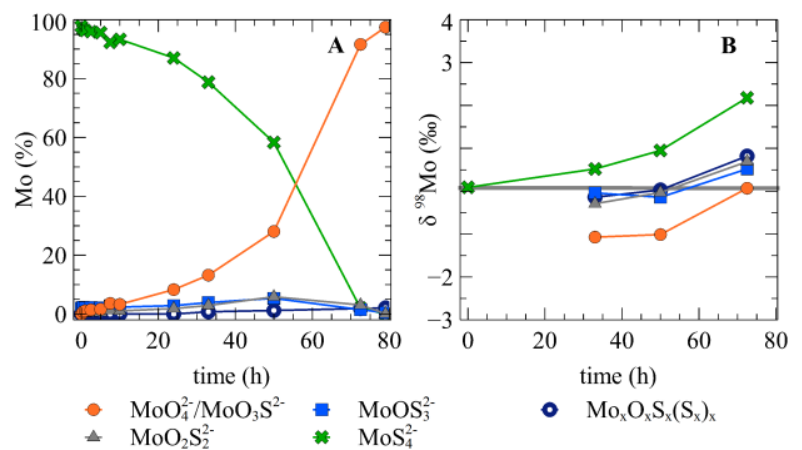

Figure 3. $200 \mu \mathrm{M} \quad \mathrm{MoS}_{4}{ }^{2-}$ transformation with 1 bar pressurized air. A: Mo species separated and analyzed with HPLC-UV in \%. B: $\delta{ }^{98}$ Mo values for Mo-species separated with HPLC-UV and isotope ratios measured with MC-ICP-MS in $\%$.

\section{CONCLUSIONS}

Here, we have presented a novel approach for direct measurement of isotope ratios in individual trace metal species co-occurring in solution, by coupling (offline) analytical chromatography to separate the individual thiomolybdates and analysis of Mo isotope ratios in the separated chromatographic fractions with MC-ICP-MS. Good reproducibility and accuracy can be achieved if complete peaks are collected to avoid isotope fractionation within peaks for species with longer retention time. Using this method, we were able to deliver the first experimental proof for isotope fractionation during formation of thiomolybdates. For $\mathrm{MoS}_{4}{ }^{2-}$ our experimental isotope fractionation nicely matches values previously calculated theoretically by Tossell ${ }^{37}$. $\mathrm{MoO}_{2} \mathrm{~S}_{2}{ }^{2-}$ was in the range previously predicted by theoretical calculations, but variation observed in the experiments likely indicates superimposed kinetic isotope fractionation. Fractionation measured during the transformation of $\mathrm{MoS}_{4}{ }^{2-}$ was all governed by kinetics. Future developments of the method should address lowering detection limits to be able to measure natural samples, e.g. by using preparative columns instead of analytical columns, and further improving reproducibility and accuracy, e.g. by minimizing preparation steps between chromatographic separation and isotope analysis or by online coupling of HPLC-MC-ICPMS.

\section{ACKNOWLEDGMENT}

We acknowledge generous funding by the German Research Foundation within the Emmy Noether program (grant \# PL 302/3-1) and grant \# PL 302/10-1. The participation of ETH Zürich in this study was supported by funding from the European Union Seventh Framework Programme under grant agreement $n^{\circ} 291823$ Marie Curie FP7-PEOPLE-2011-COFUND (The new International Fellowship Mobility Programme for Experienced Researchers in Croatia - NEWFELPRO) to EBN, and by Swiss SNF grant 200021-143262 to DV.

\section{AUTHOR INFORMATION}

\section{Corresponding Author}

*E-mail: b.planer-friedrich@uni-bayreuth.de.

\section{Notes}

The authors declare no competing financial interest.

\section{REFERENCES}

(1) J. Barling, et al., Earth Planet. Sci. Lett. 2001, 193. 447-457.

(2) G. L. Arnold, et al., Science 2004, 304. 87-90.

(3) X. Chen, et al., Nat. Commun. 2015, 6. 7142.

(4) B. Kendall, et al., Geochim. Cosmochim. Acta 2015, 156. 173193.

(5) F. T. Manheim, S. Landergren, ed. K. H. Wedepohl. Springer: Berlin, 1974, pp 42-B-1-42-O-2.

(6) Y. Nakagawa, et al., Geochem. J. 2012, 46. 131-141.

(7) R. W. Collier, Limnol. \& Oceanogr. 1985, 30. 1351-1354.

(8) J. L. Morford, S. Emerson, Geochim. Cosmochim. Acta 1999, 63. 1735-1750.

(9) S. R. Emerson, S. S. Huested, Mar. Chem. 1991, 34. 177-196. (10) P. G. Berrang, E. V. Grill, Mar. Chem. 1974, 2. 125-148.

(11) G. B. Shimmield, N. B. Price, Mar. Chem. 1986, 19. 261-280.

(12) T. F. Nägler, et al., Geostand. Geoanal. Res. 2013, 38. 149151.

(13) J. Barling, A. D. Anbar, Earth Planet. Sci. Lett. 2004, 217. 315-329.

(14) C. Siebert, et al., Earth Planet. Sci. Lett. 2003, 211. 159-171. (15) L. E. Wasylenki, et al., Geochim. Cosmochim. Acta 2008, 72. 5997-6005.

(16) C. Siebert, et al., Earth Planet. Sci. Lett. 2006, 241. 723-733.

(17) C. Archer, D. Vance, Nat. Geosci. 2008, 1. 597-600.

(18) B. E. Erickson, G. R. Helz, Geochim. Cosmochim. Acta 2000, 64. 1149-1158.

(19) G. R. Helz, et al., Geochim. Cosmochim. Acta 1996, 60. 3631-3642.

(20) T. P. Vorlicek, et al., Geochim. Cosmochim. Acta 2004, 68. 547-556.

(21) T. P. Vorlicek, G. R. Helz, Geochim. Cosmochim. Acta 2002, 66. 3679-3692.

(22) G. R. Helz, et al., Chem. Geol. 2011, 284. 323-332. 
(23) T. F. Nägler, et al., Chem. Geol. 2011, 289. 1-11.

(24) N. Neubert, et al., Geology 2008, 36. 775.

(25) A. Chappaz, et al., Geochim. Cosmochim. Acta 2014, 126.

112-122.

(26) S. E. Calvert, T. F. Pedersen, Mar. Geol. 1993, 113. 67-88.

(27) R. L. Poulson, et al., Geology 2006, 34. 617.

(28) R. L. Poulson Brucker, et al., Geochem., Geophys., Geosyst. 2009, 10. 1-25.

(29) T. Goldberg, et al., Geochim. Cosmochim. Acta 2009, 73. 6502-6516.

(30) J. McManus, et al., Geochem., Geophys., Geosyst. 2002, 3. 19.

(31) S. Westermann, et al., Earth Planet. Sci. Lett. 2014, 404. 178189.

(32) I. Azrieli-Tal, et al., Earth Planet. Sci. Lett. 2014, 393. 231242.

(33) A. D. Herrmann, et al., Chem. Geol. 2012, 324-325. 87-98.

(34) G. W. Gordon, et al., Geology 2009, 37. 535-538.

(35) A. Reitz, et al., Chem. Geol. 2007, 245. 1-8.

(36) K. Scheiderich, et al., Chem. Geol. 2010, 279. 134-144.

(37) J. A. Tossell, Geochim. Cosmochim. Acta 2005, 69. 29812993.

(38) R. Lohmayer, et al., Anal. Chem. 2015, 87. 3388-95.

(39) J. Weiss, et al., Journal of Chromatography A 1988, 439. 93108.

(40) C. Siebert, et al., Geochem., Geophys., Geosyst. 2001, 2. 116.

(41) J. Noordmann, et al., Chem. Geol. 2015, 396. 182-195.

(42) A. D. Anbar, et al., Anal. Chem. 2001, 73. 1425-1431.

(43) H. Wen, et al., J. Anal. At. Spectrom 2010, 25. 716.

(44) D. Malinovsky, F. Vanhaecke, J. Anal. At. Spectrom 2014, 29. 1090-1097.

(45) T. W. Dahl, et al., Geochim. Cosmochim. Acta 2010, 74. 144163. 
TOC

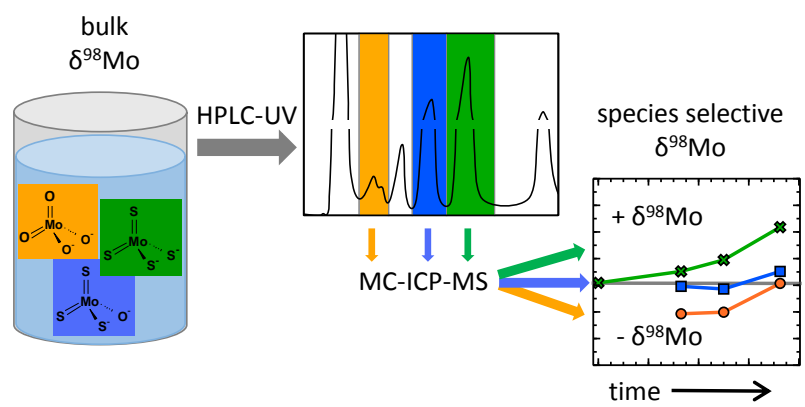

